\title{
Rotating a Rashba-coupled Fermi gas in two dimensions
}

\author{
E. Doko, ${ }^{1}$ A. L. Subaşı, ${ }^{2}$ and M. Iskin ${ }^{1}$ \\ ${ }^{1}$ Department of Physics, Koç University, Rumelifeneri Yolu, 34450 Sarlyer, Istanbul, Turkey \\ ${ }^{2}$ Department of Physics, Faculty of Science and Letters, Istanbul Technical University, 34469 Maslak, Istanbul, Turkey
}

(Received 8 October 2015; revised manuscript received 7 January 2016; published 22 March 2016)

\begin{abstract}
We analyze the interplay of adiabatic rotation and Rashba spin-orbit coupling on the BCS-BEC evolution of a harmonically trapped Fermi gas in two dimensions under the assumption that vortices are not excited. First, by taking the trapping potential into account via both the semiclassical and exact quantum-mechanical approaches, we firmly establish the parameter regime where the noninteracting gas forms a ring-shaped annulus. Then, by taking the interactions into account via the BCS mean-field approximation, we study the pair-breaking mechanism that is induced by rotation, i.e., the Coriolis effects. In particular, we show that the interplay allows for the possibility of creating either an isolated annulus of rigidly rotating normal particles that is disconnected from the central core of nonrotating superfluid pairs or an intermediate mediator phase where the superfluid pairs and normal particles coexist as a partially rotating gapless superfluid.
\end{abstract}

DOI: 10.1103/PhysRevA.93.033640

\section{INTRODUCTION}

The quantum behavior of superfluids is most evident when they are placed in a rotating container. While a slow rotation may lead to the appearance of quantized vortices or quenching of the moment of inertia [1,2], rapidly rotating systems may exhibit integer and fractional quantum Hall physics [3-6]. On the other hand, Rashba spin-orbit coupling (SOC) involves an intrinsic angular momentum, caused by coupling the particle's spin to its momentum, and it has become one of the key themes in modern condensed-matter and atomic physics, playing a central role for systems such as topological insulators and superconductors [7,8], quantum spin Hall systems [9], and spintronics applications [10].

Hence, the interplay between rotation and SOC is expected to pave the way for novel quantum phenomena, and recent realizations of SOC in atomic gases have already set the stage for such a task in a highly controllable setting [11-19]. While only the NIST-type SOC has so far been achieved, there also exist various proposals on how to create a purely Rashba SOC [20-22], the possibility of which has stimulated numerous theoretical studies on Rashba-Fermi gases in three [23-31] as well as two [32-39] dimensions. These works have revealed a plethora of intriguing phenomena, including topological superfluids, Majorana modes, spin textures, and skyrmions, which may soon be observed given the recent advances in producing a two-dimensional Fermi gas [19,40-45].

In this paper, we study the cooperation of adiabatic rotation and Rashba SOC on the ground-state phases of a trapped Fermi gas assuming that vortices are not excited. Adiabaticity requires that the rotation is introduced slowly to the system. In particular the rate of change of rotation frequency should be much smaller than the quasiparticle excitation frequency for vortex creation. In the absence of SOC, the effects of rotation on three-dimensional Fermi gases have previously been studied under this assumption at unitarity using the quantum Monte Carlo equations of state together with the local-density approximation (LDA) [46,47], and throughout the BCS-BEC evolution using the BCS mean-field approximation together with both LDA [48] and the Bogoliubov-de Gennes approach [49]. These works have shown that, by breaking some of the superfluid (SF) pairs via the Coriolis effects, rotation gives rise to a phase separation between a nonrotating SF core at the center and a rigidly rotating normal $(\mathrm{N})$ particle at the outer edge $[46,47]$, with a partially rotating gapless SF (gSF) region in between where the $\mathrm{SF}$ pairs and $\mathrm{N}$ particles coexist together $[48,49]$. Since the effects of the Coriolis force on a neutral particle in the rotating frame are similar to those of the Lorentz force on a charged particle in a magnetic field, current advances in simulating artificial fields with ultracold atoms opens alternative ways of effectively realizing a rotating Fermi gas with SOC. In addition, more recent works have confirmed that the pair-breaking scenario is energetically more favored against vortex formation in a sizable parameter regime [50,51], and experimental schemes for realizing a rotating spin-orbit-coupled system are described in Ref. [52].

In the presence of a Rashba SOC, we first take the harmonic confinement into account via both the semiclassical LDA and exact approaches and find the parameter regime where the noninteracting gas forms a ring-shaped annulus. Neither rotation nor SOC alone can deplete the central density to zero no matter how fast the rotation or how large the SOC, and the formation of such an intriguing annulus requires them both simultaneously. Then, we take the interactions into account via the BCS mean field and analyze the pair-breaking mechanism in the entire BCS-BEC evolution. In particular, we show that the cooperation of rotation and SOC allows for the possibility of creating either an isolated annulus of $\mathrm{N}$ particles that is disconnected from the central SF core or separated from it by an intermediate gSF phase as sketched in Fig. 1. We also construct phase diagrams showing the very first emergence of an $\mathrm{N}$ phase and the complete destruction of the central SF core.

\section{THEORETICAL FORMALISM}

To obtain these results, we use the following Hamiltonian density in the rotating frame:

$$
\begin{aligned}
H(\mathbf{r})= & \psi^{\dagger}(\mathbf{r})\left[\frac{\mathbf{p}^{2}}{2 M}+V(r)-\mu-\Omega L_{z}+\alpha \mathbf{p} \cdot \vec{\sigma}\right] \psi(\mathbf{r}) \\
& +\Delta(\boldsymbol{r}) \psi_{\uparrow}^{\dagger}(\mathbf{r}) \psi_{\downarrow}^{\dagger}(\mathbf{r})+\Delta^{*}(\boldsymbol{r}) \psi_{\downarrow}(\mathbf{r}) \psi_{\uparrow}(\mathbf{r})+\frac{|\Delta(\boldsymbol{r})|^{2}}{g}
\end{aligned}
$$




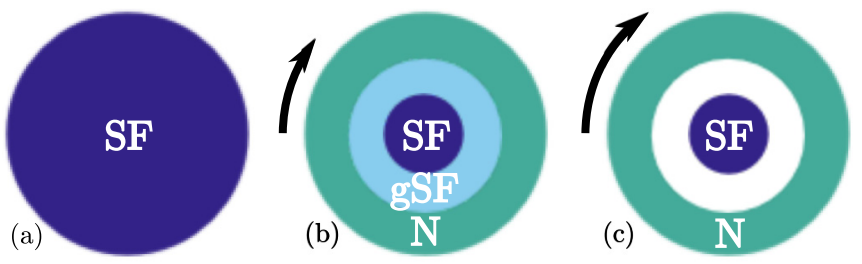

FIG. 1. Cartoon pictures showing that (a) the whole gas is a SF in the absence of rotation, (b) an intermediate gSF phase (where the $\mathrm{SF}$ and $\mathrm{N}$ particles coexist) emerges due to pair breaking induced by sufficiently high rotations, and (c) N particles are disconnected from the central SF core due to SOC at sufficiently high rotations.

$$
H_{\mathbf{k}}=\left(\begin{array}{cc}
\xi_{\mathbf{k}}-\Omega L_{\mathbf{k}} & S_{\mathbf{k}} \\
S_{\mathbf{k}}^{*} & \xi_{\mathbf{k}}-\Omega L_{\mathbf{k}} \\
0 & -\Delta^{*} \\
\Delta^{*} & 0
\end{array}\right.
$$

governs the dynamics. The index $\mathbf{r}$ is dropped here and throughout for notational simplicity. In momentum space, $\psi_{\mathbf{k}}^{\dagger}=\left(a_{\mathbf{k} \uparrow}^{\dagger}, a_{\mathbf{k} \downarrow}^{\dagger}, a_{-\mathbf{k} \uparrow}, a_{-\mathbf{k} \downarrow}\right)$ and $a_{\mathbf{k}, \sigma}\left(a_{\mathbf{k}, \sigma}^{\dagger}\right)$ annihilates (creates) a $\sigma$ fermion with momentum $\mathbf{k}=\left(k_{x}, k_{y}\right)$. The freeparticle dispersion relative to the local chemical potential $\mu_{\mathbf{r}}=$ $\mu-V(r)$ is $\xi_{\mathbf{k}}=\epsilon_{\mathbf{k}}-\mu_{\mathbf{r}}$ with $\epsilon_{\mathbf{k}}=k^{2} /(2 M)$. The rotation enters via $\Omega L_{\mathbf{k}}=\mathbf{v}(\mathbf{r}) \cdot \mathbf{k}$ with the velocity $\mathbf{v}(\mathbf{r})=\Omega \hat{\mathbf{z}} \times$ $\mathbf{r}, S_{\mathbf{k}}=\alpha\left(k_{x}-i k_{y}\right)$ is the SOC term, $\Delta=g \sum_{\mathbf{k}}\left\langle a_{\mathbf{k} \uparrow} a_{-\mathbf{k} \downarrow}\right\rangle$ denotes the SF order parameter, and $C=\sum_{\mathbf{k}}\left(\xi_{\mathbf{k}}+\Omega L_{\mathbf{k}}\right)+$ $|\Delta|^{2} / g$ is a constant.

We diagonalize Eq. (2) and express $H_{\text {loc }}=C+$ $\sum_{\mathbf{k} s}\left(E_{\mathbf{k} s} \gamma_{\mathbf{k} s}^{\dagger} \gamma_{\mathbf{k} s}-E_{\mathbf{k} s} / 2\right)$, where $\gamma_{\mathbf{k} s}^{\dagger}\left(\gamma_{\mathbf{k} s}\right)$ creates (annihilates) a quasiparticle with helicity $s= \pm$ and energy $E_{\mathbf{k} s}=$ $\sqrt{\left(\xi_{\mathbf{k}}+s \alpha k\right)^{2}+|\Delta|^{2}}-\Omega L_{\mathbf{k}}$. Thus, the thermodynamic potential $G=-(1 / \beta) \operatorname{Tr}\left(\ln e^{-\beta H_{\mathrm{loc}}}\right)$, where $\beta=1 /\left(k_{B} T\right)$ with $k_{B}$ the Boltzmann constant and $T$ the temperature, can be written as $G=C+\sum_{\mathbf{k} s}\left[E_{\mathbf{k} s} f\left(E_{\mathbf{k} s}\right)-E_{\mathbf{k} s} / 2\right]$ with the Fermi function $f(x)=1 /\left(e^{\beta x}+1\right)$. Setting $\partial G / \partial|\Delta|=0$ and using $n(\mathbf{r})=-(1 / A) \partial G / \partial \mu_{\mathbf{r}}$ for the local particle density in area $A$, we finally obtain the local self-consistency equations,

$$
\begin{gathered}
\frac{1}{g}=\frac{1}{4} \sum_{\mathbf{k} s} \frac{1}{E_{\mathbf{k} s}+\Omega L_{\mathbf{k}}} \tanh \left(\beta E_{\mathbf{k} s} / 2\right), \\
n(\mathbf{r})=\frac{1}{2 A} \sum_{\mathbf{k} s}\left[1-\frac{\xi_{\mathbf{k}}+s \alpha k}{E_{\mathbf{k} s}+\Omega L_{\mathbf{k}}} \tanh \left(\beta E_{\mathbf{k} s} / 2\right)\right],
\end{gathered}
$$

such that the total number of particles is given by $N=$ $\int d \mathbf{r} n(\mathbf{r})$. These equations are the generalizations of the usual BCS expressions to the case of rotation and SOC, and it is a standard practice to substitute the two-body binding energy in vacuum $E_{b} \geqslant 0$ for $g$ via the relation $1 / g=\sum_{\mathbf{k}} 1 /\left(2 \epsilon_{\mathbf{k}}+E_{b}\right)$ in the cold-atom context. While a nonzero (vanishing) $\Delta$ is a characteristic of the SF (N) phase in general, we also use the mass-current density $\mathbf{J}=\left(J_{x}, J_{y}\right)$ flowing in the azimuthal direction, where

$$
\left(J_{x}, J_{y}\right)=\frac{1}{A} \sum_{\mathbf{k}}\left[n_{\mathbf{k}} \mathbf{k}+2 M \alpha\left(\operatorname{Re}\left\{p_{\mathbf{k}}\right\}, \operatorname{Im}\left\{p_{\mathbf{k}}\right\}\right)\right],
$$

where $\psi^{\dagger}=\left(\psi_{\uparrow}^{\dagger}, \psi_{\downarrow}^{\dagger}\right)$ denotes the field operators, $\mathbf{p}=-i \nabla$ is the linear-momentum operator with $\hbar=1, V(r)=M \omega^{2} r^{2} / 2$ is the trapping potential, $\mu$ is the chemical potential, $0 \leqslant$ $\Omega<\omega$ is the rotation frequency, $L_{z}$ is the $z$ projection of the angular-momentum operator $\mathbf{L}=\mathbf{r} \times \mathbf{p}, \alpha \geqslant 0$ is the strength of the Rashba coupling, $\vec{\sigma}=\left(\sigma_{x}, \sigma_{y}\right)$ is a vector of Pauli spin matrices, and $\Delta(\boldsymbol{r})=g\left\langle\psi_{\uparrow}(\mathbf{r}) \psi_{\downarrow}(\mathbf{r})\right\rangle$ is the mean-field SF order parameter with $g \geqslant 0$ being the strength of attractive interactions and $\langle\cdots\rangle$ the thermal average. Within the semiclassical LDA, the local Hamiltonian can be written as $H_{\text {loc }}=(1 / 2) \sum_{\mathbf{k}} \psi_{\mathbf{k}}^{\dagger} H_{\mathbf{k}} \psi_{\mathbf{k}}+C$, where the matrix

$$
\left.\begin{array}{cc}
0 & \Delta \\
-\Delta & 0 \\
-\xi_{\mathbf{k}}-\Omega L_{\mathbf{k}} & S_{\mathbf{k}}^{*} \\
S_{\mathbf{k}} & -\xi_{\mathbf{k}}-\Omega L_{\mathbf{k}}
\end{array}\right)
$$

to further characterize the gSF phase. Here, $n_{\mathbf{k}}=\left\langle a_{\mathbf{k} \uparrow}^{\dagger} a_{\mathbf{k} \uparrow}\right\rangle+$ $\left\langle a_{\mathbf{k} \downarrow}^{\dagger} a_{\mathbf{k} \downarrow}\right\rangle$ is the momentum distribution given by the summand of Eq. (4), Re and Im denote the real and imaginary parts, and $p_{\mathbf{k}}=\left\langle a_{\mathbf{k} \uparrow}^{\dagger} a_{\mathbf{k} \downarrow}\right\rangle$. Let us first set $\Delta=0$ and $T=0$ and analyze the noninteracting ground state.

\section{NONINTERACTING PROBLEM}

In the absence of both rotation and SOC, the noninteracting gas has the shape of a disk with its density $n(r)$ decreasing parabolically as a function of $r$, up to the edge of the system given by the Thomas-Fermi radius $R_{F}=\sqrt{2 E_{F} /\left(M \omega^{2}\right)}$, where $E_{F}=k_{F}^{2} /(2 M)=\omega \sqrt{N}$ is the Fermi energy. In the presence of rotation only, while the gas retains its overall parabolic density, some of the particles are expelled from the center of the trap due to the centrifugal effects, and the edge moves to $\widetilde{R}_{F}=R_{F} /\left(1-\Omega^{2} / \omega^{2}\right)^{1 / 4}$. However, in the presence of SOC only, $n(r)$ tends to increase at the center and the gas shrinks due to the increased low-energy density of states. Therefore, $\Omega \neq 0$ and $\alpha \neq 0$ have counteracting effects on $n(r)$ in general. In addition, since $\Omega \neq 0$ causes an asymmetry in the local $\mathbf{k}$ space and $\alpha \neq 0$ shifts its dispersion minima from $\mathbf{k}=\mathbf{0}$ to $\mathbf{k} \neq \mathbf{0}$, their interplay is expected to give rise to a much richer physics even in the noninteracting limit. For instance, setting $\Delta=0$ in Eq. (4), and solving for $n(r)=0$, we find an analytic expression for the edge(s) of the gas given by

$$
R_{O, I}^{0}=R_{F} \frac{\omega \Omega \alpha \pm \omega \sqrt{\alpha^{2} \omega^{2}+2 \mu\left(\omega^{2}-\Omega^{2}\right) / M}}{k_{F}\left(\omega^{2}-\Omega^{2}\right) / M},
$$

where $R_{O}^{0}\left(R_{I}^{0}\right)$ is the radius of the outer (inner) edge. Note that while $R_{O}^{0}$ is positive and exists for all parameters as long as $\Omega<\omega$, a positive $R_{I}^{0}$ is possible only for the parameter regimes where $\alpha^{2}+2 \mu / M<0$. While the gas has the usual shape of a disk with $n(r) \neq 0$ for $0 \leqslant r<R_{O}^{0}$ when $\alpha^{2}>-2 \mu / M$, otherwise it has the shape of a ring with $n(r) \neq 0$ in the radial interval $R_{I}^{0}<r<R_{O}^{0}$. 

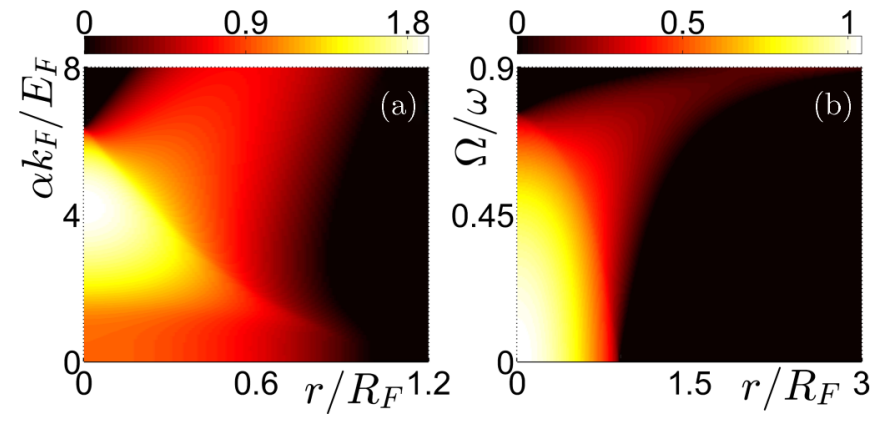

FIG. 2. Noninteracting $n(r)$ profiles [in units of $n_{0}=k_{F}^{2} /(2 \pi)$ ] are shown as a function of (a) $\alpha$ for $\Omega=0.15 \omega$ and (b) $\Omega$ for $\alpha=$ $1 E_{F} / k_{F}$. The gas eventually forms a ring-shaped annulus in both figures.

The formation of such an annulus can also be illustrated by solving $n(r)$ directly from Eq. (4), which we present in Fig. 2. As shown in Fig. 2(a), while increasing $\alpha$ initially increases $n(r)$ near the central region due to the increase in the low-energy density of states, the $\mathbf{k} \neq \mathbf{0}$ states gain further energy through their angular momentum in the rotating frame, and $n(r)$ decreases dramatically away from the center as $\alpha$ increases further. As the critical condition is approached, the latter effect gradually dominates, causing the central density to decrease as the gas continues to expand, and $n(0)$ eventually vanishes beyond $\alpha=\sqrt{-2 \mu / M}$. We also see a similar behavior in Fig. 2(b), where increasing $\Omega$ is shown to deplete $n(0)$ to zero once the critical condition is satisfied. This happens at faster $\Omega$ for smaller $\alpha$ and vice versa. In contrast to increasing $\alpha$, however, increasing $\Omega$ not only decreases $n(0)$ but also expands the gas monotonically. It is important to emphasize here that neither rotation nor SOC alone can deplete $n(0)$ to zero no matter how fast $\Omega$ or large $\alpha$ is, and the formation of an annulus requires them both simultaneously.

In the noninteracting limit, we benchmark our semiclassical results with those of exact quantum-mechanical treatment and find an excellent agreement between the two for all parameters including the fast- $\Omega$ and/or large- $\alpha$ limits. The details of this comparison are given in the Appendix. Motivated by this success, next we apply the LDA formalism to the entire BCS-BEC evolution at $T=0$.

\section{INTERACTING PROBLEM}

In the absence of both rotation and SOC, the superfluid persists with $\Delta \neq 0$ as long as $n(r) \neq 0$ and, therefore, the entire system is a disk-shaped gapped SF with its edge located at $R_{O}=R_{F}$ no matter how strong or weak $E_{b}$ is, which only happens in two dimensions. In the presence of an adiabatic rotation, since vortices are assumed not to be excited in the system and the gapped SF cannot carry the angular momentum, some of the SF pairs must eventually break by the centrifugal effects, i.e., via the broken time-reversal symmetry, giving rise to $\mathrm{gSF}$ and $\mathrm{N}$ regions in the trap. In this paper, we distinguish gSF from SF by checking whether $J$ is nonzero or not, or equivalently whether $E_{\mathbf{k} s}$ has negative regions in $\mathbf{k}$ space or not. In addition, while both gSF and N phases have $J \neq 0$, only the $\mathrm{N}$ region rotates rigidly as a whole with $J=M n(r) \Omega r$.

When $\alpha=0$, the robustness of the SF pairs against rotation depends on $E_{b}$ and $\Omega$ in such a way that there is no pair
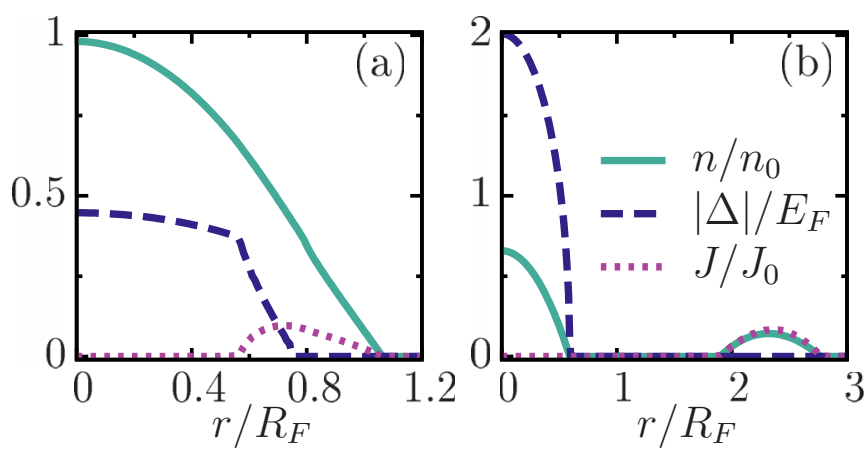

FIG. 3. Typical profiles showing (a) a partially rotating gSF region and (b) a vacuum separating the nonrotating SF pairs from the rigidly rotating $\mathrm{N}$ particles. Here, $E_{b}=0.1 E_{F}, \alpha=0.8 E_{F} / k_{F}$, and $\Omega=0.3 \omega$ in (a), and $E_{b}=1 E_{F}, \alpha=7 E_{F} / k_{F}$, and $\Omega=0.5 \omega$ in (b), where $n_{0}=k_{F}^{2} /(2 \pi)$ and $J_{0}=M n_{0} \omega R_{F}$.

breaking when $\Omega$ is sufficiently slow for a given $E_{b}$. Therefore, $n(r), \Delta$, and $\mu$ are not affected by rotation as long as $\Omega<\Omega_{c}$, and the entire system is again a disk-shaped SF with its edge located at $R_{O}=R_{F}$. We determine $\Omega_{c}$ by noting that when the first broken pair emerges as $\mathrm{N}$ particles at the edge of the gas then its radius must coincide with the Thomas-Fermi radius of the nonrotating gas with the same $\mu$, i.e., $R_{O}^{0}(\alpha \rightarrow 0, \Omega \rightarrow$ $\left.\Omega_{c}\right)=R_{F}$, leading to $\Omega_{c}=\omega \sqrt{E_{b} /\left(2 E_{F}\right)}$. Since $\Omega<\omega$ has a physical upper bound in order not to lose the particles from the harmonic trap, the SF pairs are perfectly robust against rotation for $E_{b}>2 E_{F}$ in the $\alpha \rightarrow 0$ limit. When $\Omega>\Omega_{c}$, the trap profile in general consists of three distinct regions: while the central (outer) core (wing) is solely occupied by paired (unpaired) $\mathrm{SF}(\mathrm{N})$ particles, the SF pairs and $\mathrm{N}$ particles coexist in the middle, a result of which is that the gSF emerges as an intermediate phase around the SF-N interface. We note that, even though the SF core shrinks monotonically and gives way to the $\mathrm{N}$ phase with increasing $\Omega$, it still survives in the $\Omega \rightarrow \omega$ limit.

The preceding description still holds when $\alpha \neq 0$ but small, and we illustrate a typical trap profile in Fig. 3(a), where a small gSF region is clearly visible at the SF-N interface. Similar to the $\alpha=0$ case, the critical rotation frequency for the emergence of $\mathrm{N}$ particles can be determined from $R_{O}^{0}\left(\alpha, \Omega_{c}\right)=R_{O}(\alpha, \Omega=0)$. Away from the small- $\alpha$ limit, however, we find a very intriguing situation provided that $\alpha^{2}<-2 \mu / M$. For instance, a typical trap profile for this case is shown in Fig. 3(b), where the $\mathrm{N}$ particles form an isolated annulus that is completely disconnected from the central SF core without a gSF region in between. The $\Omega_{c}$ threshold for the emergence of an isolated $\mathrm{N}$ phase can be determined from $R_{O}^{0}\left(\alpha, \Omega_{c}\right)>R_{O}(\alpha, \Omega=0)$.

By repeating this analysis numerous times for a wide range of parameters, we construct the phase diagram of the system based on the very first emergence of $\mathrm{N}$ particles with increasing $\Omega$. The diagram shown in Fig. 4(a) is one of our primary results in this paper and should be read as follows. For a given $\alpha$ shown on the horizontal axis, increasing $\Omega$ in the vertical direction breaks SF pairs beyond the intersection with the $\Omega_{c}$ curve. This diagram intuitively suggests that $\Omega_{c}$ increases with increasing $E_{b}$ for a given $\alpha$. More importantly, it also shows that, in contrast to the $\alpha \rightarrow 0$ limit where SF pairs 

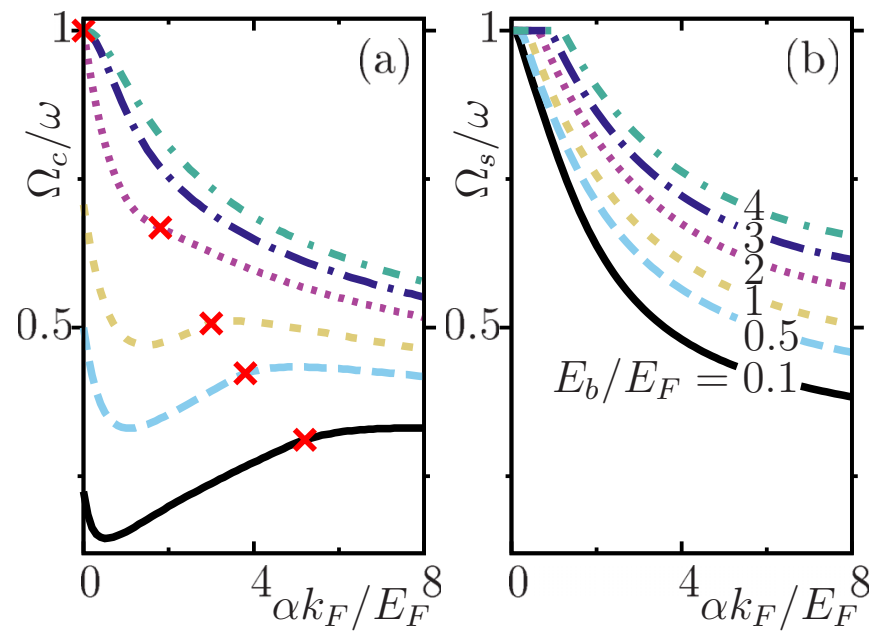

FIG. 4. Critical $\Omega$ curves are shown for (a) the emergence of an $\mathrm{N}$ phase and (b) the depletion of the SF core. Beyond the (red) crosses in (a), the $\mathrm{N}$ particles form an isolated annulus that is disconnected from the central SF core without a gSF region in between.

are perfectly robust against $\Omega<\omega$ when $E_{b}>2 E_{F}$, finite $\alpha$ eventually allows $\Omega$ to create an $\mathrm{N}$ phase for any $E_{b}$ with $\Omega_{c}<\omega$. Furthermore, depending on whether the intersection with the $\Omega_{c}$ curve is to the left or to the right of the (red) crosses, we know whether an intermediate gSF phase exists or not at the SF-N interface. In the former case, the gSF phase may eventually disappear with further increase in $\Omega$, so that the $\mathrm{N}$ phase ends up again being disconnected from the $\mathrm{SF}$ core (not shown in the phase diagram). Thus, the gSF phase always emerges for any $E_{b}<2 E_{F}$ in the $\alpha \rightarrow 0$ limit, and the $\mathrm{N}$ particles form an isolated annulus practically for any $E_{b} \gtrsim 2 E_{F}$ as long as $\alpha \neq 0$. We note that increasing $E_{b}$ moves the crosses to lower $\alpha$ because faster $\Omega_{c}$ leads to an annulus of $\mathrm{N}$ particles at smaller $\alpha$, as discussed for the noninteracting problem.

It is also possible to obtain an analytic expression for the small- $\alpha$ dependence of $\Omega_{c}$ on the left side of the crosses, by again noting that the first broken pair emerges as $\mathrm{N}$ particles at the edge $r \rightarrow R_{O}$ of the gas. We evaluate the gapless condition $E_{\mathbf{k} s}=0$ with $\Delta \rightarrow 0$, after setting $\mu \simeq$ $E_{F}-E_{b} / 2-M \alpha^{2}$ and $R_{O} \approx R_{F}$ at the lowest orders in $\alpha$, leading to $\Omega_{c} \approx \omega\left(\sqrt{2 \alpha^{2} k_{F}^{2} / E_{F}^{2}+2 E_{b} / E_{F}}-\alpha k_{F} / E_{F}\right) / 2$. This expression shows that $\Omega_{c}$ decreases with $\alpha$ at the lowest order, and it is in excellent agreement with all of our numerical results. Physically, since $\alpha \neq 0$ shifts the excitation minima to higher momenta, the lowest-energy states become more susceptible to rotation, making it easier for $\Omega$ to break the SF pairs at least in the small- $\alpha$ limit. However, followed by a sudden decrease, Fig. 4(a) also shows that the $\Omega_{c}$ curve develops a minimum as a function of $\alpha$ when $E_{b} \lesssim 2 E_{F}$. This is because of a competing mechanism where increasing $\alpha$ not only enhances $\Delta$ but also decreases $R_{O}$ by increasing $n(r)$ near the center, both of which make it more difficult for $\Omega$ to break the SF pairs away from the small- $\alpha$ limit. We also note that increasing $E_{b}$ moves the location of the minimum to larger $\alpha$ because it is only then that the competing effects caused by SOC become comparable to the enhancement of $\Delta$ caused by stronger $E_{b}$.
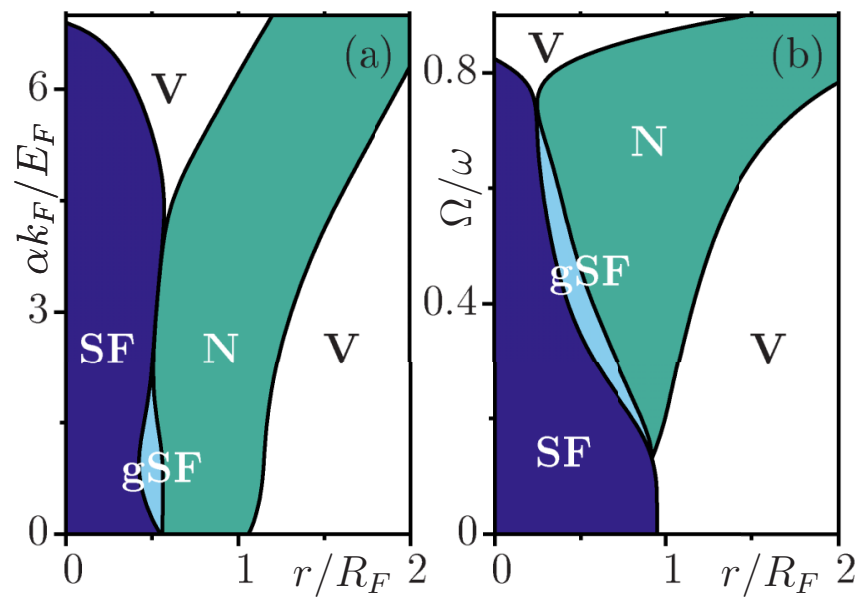

FIG. 5. Radial phase profiles are shown as a function of (a) $\alpha$ for $E_{b}=0.1 E_{F}$ and $\Omega=0.4 \omega$ and (b) $\Omega$ for $E_{b}=0.1 E_{F}$ and $\alpha=$ $0.4 E_{F} / k_{F}$. The cooperation of $\Omega$ and $\alpha$ not only destroys the central SF core but also boosts the gSF region in both figures.

Next to Fig. 4(a), we present another phase diagram showing the destruction of the central SF core under more extreme parameter regimes. For instance, typical phase profiles for this case are illustrated in Fig. 5, where the interplay of fast $\Omega$ and/or large $\alpha$ eventually dominates the effects of $E_{b} \neq 0$ and depletes $n(0)$ to zero, recovering the noninteracting problem discussed above. The diagram shown in Fig. 4(b) is also one of our primary results in this paper, where the $\Omega_{s}$ threshold for the complete destruction of the SF core is determined by first setting $\Delta \rightarrow 0$ as $r \rightarrow 0$ in Eq. (3) to get $\mu$ and then plugging these values to Eq. (4). This diagram intuitively suggests that $\Omega_{s}$ increases with increasing $E_{b}$ for a given $\alpha$. More importantly, it also shows that, in contrast to the $\alpha \rightarrow 0$ limit where the central SF pairs are robust against $\Omega<\omega$ for any $E_{b}$ since the centrifugal effects strictly vanish at $r=0$, increasing $\alpha$ eventually allows $\Omega$ to destroy all of the SF pairs.

\section{CONCLUSIONS}

To summarize, here we studied the cooperation of adiabatic rotation and Rashba SOC on the ground-state phases of a trapped Fermi gas in two dimensions, assuming that vortices are not excited. First, by taking the harmonic confinement into account via both the LDA and exact approaches, we found the parameter regime where the noninteracting gas forms a ring-shaped annulus. It is important to emphasize that neither rotation nor SOC alone can deplete the central density to zero no matter how fast the rotation or large SOC is, and the formation of such an intriguing annulus requires them both simultaneously. Then, by taking the interactions into account via the BCS mean field, we analyzed the rotation-induced pair-breaking mechanism in the entire BCS-BEC evolution. In particular, we showed that the cooperation of rotation and SOC allows for the possibility of creating either an isolated annulus of rigidly rotating $\mathrm{N}$ particles that is disconnected from the central core of nonrotating SF pairs or an intermediate gapless SF phase which is characterized by the coexistence of SF pairs and $\mathrm{N}$ particles. We also constructed phase diagrams showing 
the very first emergence of an $\mathrm{N}$ phase and the complete destruction of the central SF core. We hope that, given the ongoing push towards simulating Rashba-coupled Fermi gases by many groups worldwide, our compelling results may soon be realized once the technical experimental difficulties are cleared out of the way.

\section{ACKNOWLEDGMENTS}

This work is supported by TÜBITAK Grant No. 1001114F232 and E.D. is supported by the TÜBITAK-2215 Ph.D. Fellowship.

\section{APPENDIX: EXACT NUMERICAL SOLUTION FOR SEC. III}

In the rotating frame, the noninteracting Hamiltonian for a harmonically trapped Fermi gas with Rashba SOC can be written as

$$
H_{0}=\int d \mathbf{r} \psi^{\dagger}(\mathbf{r})\left[H_{\mathrm{HO}}-\Omega L_{z}-\mu+\alpha \mathbf{p} \cdot \vec{\sigma}\right] \psi(\mathbf{r}),
$$

where $H_{\mathrm{HO}}=\mathbf{p}^{2} /(2 M)+M \omega^{2} r^{2} / 2$ is the usual twodimensional harmonic-oscillator Hamiltonian. We make use of the rotational symmetry of the system and expand the field operators in terms of the angular-momentum basis of the two-dimensional harmonic oscillator as

$$
\psi_{\sigma}(\mathbf{r})=\sum_{n, l} g_{n, l}(\mathbf{r}) c_{n, l, \sigma},
$$

where $c_{n, l, \sigma}$ annihilates a spin- $\sigma$ particle in the $|n, l\rangle$ state that is given by the normalized real-space wave function

$$
g_{n, l}(\mathbf{r})=i^{2 n_{<}} \sqrt{\frac{n_{<} !}{\pi a_{0}^{2} n_{>} !}} e^{i l \theta} \tilde{r}^{|l|} e^{-\tilde{r}^{2} / 2} L_{n_{<}}^{|l|}\left(\tilde{r}^{2}\right),
$$

where $\tilde{r}=r / a_{0}$ with the harmonic-oscillator length $a_{0}=$ $\sqrt{1 /(M \omega)}$ scale, the energy and angular-momentum quantum numbers $n \geqslant|l| \geqslant 0$ are integers, $n_{<}\left(n_{>}\right)$is the lesser (greater) of $(n \pm l) / 2$, and $L_{k}^{|m|}(x)=\left(x^{-|m|} e^{x} / k !\right) d^{k}\left(e^{-x} x^{k+|m|}\right) / d x^{k}$ is the $k$ th-degree associated Laguerre polynomial. The noninteracting Hamiltonian can be written in this basis as

$$
\begin{aligned}
H_{0}= & \sum_{n, l, \sigma}[\omega(n+1)-\Omega l-\mu] c_{n, l, \sigma}^{\dagger} c_{n, l, \sigma} \\
& +\frac{\alpha}{2 a_{0}} \sum_{n, l}\left(i \sqrt{\frac{n+l}{2}+1} c_{n+1, l+1, \downarrow}^{\dagger} c_{n, l, \uparrow}\right. \\
& \left.-i \sqrt{\frac{n-l}{2}} c_{n-1, l+1, \downarrow}^{\dagger} c_{n, l, \uparrow}+\text { H.c. }\right),
\end{aligned}
$$

where H.c. is the Hermitian conjugate. Even though the Rashba coupling $\alpha \mathbf{p} \cdot \vec{\sigma}$ does not preserve the individual spin or real-space rotational symmetry, the full rotational symmetry is still manifest. Since $H_{0}$ commutes with the total angular-momentum operator $J_{z}=L_{z}+S_{z}=$ $\sum_{n, l, \sigma}\left(l+\frac{\sigma_{z}}{2}\right) c_{n, l, \sigma}^{\dagger} c_{n, l, \sigma}$, where $\sigma_{z}= \pm 1$ for $\sigma=(\uparrow, \downarrow)$, respectively, they can be simultaneously diagonalized.

Using the conservation of $J_{z}$, the Hamiltonian can be expressed in a block-diagonal form, $H_{0}=\sum_{l} \Psi_{l}^{\dagger} H_{l} \Psi_{l}$, where $H_{l}$ is a tridiagonal matrix in each block with the following ordering of the basis states. For $l \geqslant 0$ corresponding to the $J_{z}$ subspace $(l \uparrow, l+1 \downarrow)$ with eigenvalue $j=l+1 / 2>0, H_{l}$ is given by

$$
\left(\begin{array}{cccccc}
\epsilon_{l, l} & a \sqrt{l+1} & & & & \\
a^{*} \sqrt{l+1} & \epsilon_{l+1, l+1} & a \sqrt{1} & & & \\
& a^{*} \sqrt{1} & \epsilon_{l+2, l} & a \sqrt{l+2} & & \\
& & a^{*} \sqrt{l+2} & \epsilon_{l+3, l+1} & a \sqrt{2} & \\
& & & a^{*} \sqrt{2} & \epsilon_{l+4, l} & \ddots \\
& & & & \ddots & \ddots
\end{array}\right),
$$

where the basis vectors are ordered as $\Psi_{l}^{\dagger}=\left(c_{n=l, l, \uparrow}^{\dagger}, c_{l+1, l+1, \downarrow}^{\dagger}, c_{l+2, l, \uparrow}^{\dagger}, c_{l+3, l+1, \downarrow}^{\dagger}, \ldots\right)$. Here, $\epsilon_{n, l}=\omega(n+1)-\Omega l-\mu$ and $a=i \alpha / 2 a_{0}$. Similarly for $l<0$ corresponding to the $(-|l|+1 \downarrow,-|l| \uparrow)$ subspace with eigenvalue $j=l+1 / 2<0, H_{l}$ is given by

$$
\left(\begin{array}{cccccc}
\epsilon_{-l-1, l+1} & a \sqrt{-l} & & & & \\
a^{*} \sqrt{-l} & \epsilon_{-l, l} & a \sqrt{1} & & & \\
& a^{*} \sqrt{1} & \epsilon_{-l+1, l+1} & a \sqrt{-l+1} & & \\
& & a^{*} \sqrt{-l+1} & \epsilon_{-l+2, l} & a \sqrt{2} & \\
& & & a^{*} \sqrt{2} & \epsilon_{-l+3, l+1} & \ddots \\
& & & & \ddots & \ddots
\end{array}\right),
$$


where the basis vectors are ordered as $\Psi_{l}^{\dagger}=$ $\left(c_{n=-l-1, l+1, \downarrow}, c_{-l, l, \uparrow}, c_{-l+1, l+1, \downarrow}, c_{-l+2, l, \uparrow}, \ldots\right)$. This Hamiltonian can be diagonalized via the unitary transformation $\quad c_{n, l, \sigma}=\sum_{m} u_{n \sigma, m}^{(j)} C_{j, m}$, leading to $H_{0}=\sum_{j, m} E_{m}^{(j)} C_{j, m}^{\dagger} C_{j, m}$, where $j=l+\sigma_{z} / 2$ and the $\left\{u_{n \sigma, m}^{(j)}\right\}$ is the eigenvector characterizing the $m$ th energy eigenstate in the $j=l+1 / 2$ subspace.

Note that this particular form of the Hamiltonian is similar to that of a Bogoliubov-de Gennes Hamiltonian of a harmonically trapped spinless $p$-wave superconductor [53]. Furthermore, the complex number $i$ in the complex factor $a$ can be absorbed in the even-numbered eigenvector components so that numerically a tridiagonal symmetric matrix can be diagonalized. In practice, a cutoff energy $E_{c}=\omega\left(l_{\max }+\right.$ 1) $\gg E_{F}$ is introduced and finite matrices are diagonalized with basis states having less energy than the cutoff. We checked that our numerical results for the the low-energy eigenvalues and eigenvectors are independent of the chosen cutoff.

Since SOC couples $\uparrow$ and $\downarrow$ spins, the mass-current density should be identified from the continuity equation

$$
\partial_{t} \rho(\mathbf{r})+\nabla \cdot \mathbf{J}(\mathbf{r})=0
$$

where $\rho(\mathbf{r})=\operatorname{Mn}(\mathbf{r})$ is the mass density. The expectation value of the density and mass-current density operators are given as

$$
\begin{gathered}
n(r)=\sum_{\sigma}\left\langle\psi_{\sigma}^{\dagger}(r) \psi_{\sigma}(r)\right\rangle=\sum_{n, n^{\prime}, l, l^{\prime}, \sigma} g_{n, l}^{*}(r) g_{n^{\prime}, l^{\prime}}(r)\left\langle c_{n, l, \sigma}^{\dagger} c_{n^{\prime}, l^{\prime}, \sigma}\right\rangle \\
=\sum_{j} \sum_{m}\left[n_{\uparrow, m}^{(j)}(r)+n_{\downarrow, m}^{(j)}(r)\right] f\left[E_{m}^{(j)}\right], \\
J_{\theta}(r)=\sum_{\sigma} \operatorname{Im}\left\langle\psi_{\sigma}^{\dagger}(r) \frac{\partial}{r \partial \theta} \psi_{\sigma}(r)\right\rangle+2 M \alpha\left|\left\langle\psi_{\uparrow}^{\dagger}(r) \psi_{\downarrow}(r)\right\rangle\right| \\
=M \sum_{j} \sum_{m} J_{\theta, m}^{(j)}(r) f\left[E_{m}^{(j)}\right],
\end{gathered}
$$

where $f\left[E_{m}^{(j)}\right]=\left\langle C_{j, m}^{\dagger} C_{j, m}\right\rangle$ with $\langle\cdots\rangle$ the thermal average and $f(x)=1 /\left(e^{\beta x}+1\right)$ is the Fermi-Dirac distribution, and Im denotes the imaginary part. Here, the density $n_{\sigma, m}^{(j)}(r)$ and the angular component of the mass current density $J_{\theta, m}^{(j)}(r)$ for the $m$ th energy eigenstate with total angular momentum $j$ are given by

$$
\begin{gathered}
n_{\uparrow, m}^{(j)}(r)=\left|\sum_{k=0}^{n_{c} / 2} u_{l+2 k \uparrow, m}^{(j)} g_{l+2 k, l}(r, \theta)\right|^{2}, \\
n_{\downarrow, m}^{(j)}(r)=\left|\sum_{k=0}^{n_{c} / 2} u_{l+1+2 k \downarrow, m}^{(j)} g_{l+2 k, l+1}(r, \theta)\right|^{2},
\end{gathered}
$$

$$
\begin{aligned}
J_{\theta, m}^{(j)}(r)= & \frac{l}{r} n_{\uparrow, m}^{(j)}(r)+\frac{l+1}{r} n_{\downarrow, m}^{(j)}(r) \\
& +2 \alpha \mid\left[\sum_{k=0}^{n_{c} / 2} u_{l+2 k \uparrow, m}^{(j)} g_{l+2 k, l}(r, \theta)\right] \\
& \times\left\lfloor\sum_{k=0}^{n_{c} / 2} u_{l+1+2 k \downarrow, m}^{(j)} g_{l+2 k, l+1}(r, \theta)\right],
\end{aligned}
$$

for $j>0(l \geqslant 0)$ and

$$
\begin{aligned}
n_{\uparrow, m}^{(j)}(r) & =\left|\sum_{k=0}^{n_{c} / 2} u_{-l+2 k \uparrow, m}^{(j)} g_{l+2 k, l}(r, \theta)\right|^{2}, \\
n_{\downarrow, m}^{(j)}(r) & =\left|\sum_{k=0}^{n_{c} / 2} u_{-l-1+2 k \downarrow, m}^{(j)} g_{l+2 k, l+1}(r, \theta)\right|^{2}, \\
J_{\theta, m}^{(j)}(r)= & \frac{l}{r} n_{\uparrow, m}^{(j)}(r)+\frac{l+1}{r} n_{\downarrow, m}^{(j)}(r) \\
& +2 \alpha \mid\left[\sum_{k=0}^{n_{c} / 2} u_{-l+2 k \uparrow, m}^{(j)} g_{l+2 k, l}(r, \theta)\right]^{*} \\
& \times\left[\sum_{k=0}^{n_{c} / 2} u_{-l-1+2 k \downarrow, m}^{(j)} g_{l+2 k, l+1}(r, \theta)\right]
\end{aligned}
$$

for $j<0 \quad(l<0)$ and $n_{c}=\left(|l|_{\max }-|l|\right) / 2$. The density is independent of the angle $\theta$ and the radial component of the mass-current density is zero for the energy eigenstates.

In Fig. 6, we present the number and mass-current density profiles in the trap that are obtained from the exact calculation given above and the LDA approach described in the main text, showing an excellent agreement between the two. The total number of particles is $N=2 \pi \int d r r n(r)=800$. The finite-size effects vanish in the thermodynamic limit when $N \rightarrow \infty$.

In comparing the exact quantum-mechanical calculations with LDA, we scale the radial distance by the Thomas-Fermi radius $R_{F}$ and the density by $n_{0}=k_{F}^{2} /(2 \pi)$, which are defined via $E_{F}=k_{F}^{2} /(2 M)=M \omega^{2} R_{F} / 2$. Here, the Fermi energy is defined for the nonrotating gas in the absence of SOC as $E_{F}=\omega \sqrt{N}$ in LDA, so that $\left(k_{F} a_{0}\right)^{2}=2 \sqrt{N}$ and $R_{F} / a_{0}=$ $(4 N)^{1 / 4}$. In this case, the exact quantum-mechanical solution gives $E_{F}=\omega\left(N_{F}+1\right)$ and $N=\left(N_{F}+1\right)\left(N_{F}+2\right)$ with the quantum number $N_{F}$. This is consistent with the LDA result $E_{F}=\omega \sqrt{N}$ for a large number of particles, $N$, in the trap such that $N_{F} \gg 1$. The mass-current density is in units of $J_{0}=M n_{0} \omega R_{F}=\sqrt{2} N^{3 / 4} /\left(\pi a_{0}^{3}\right)$. 

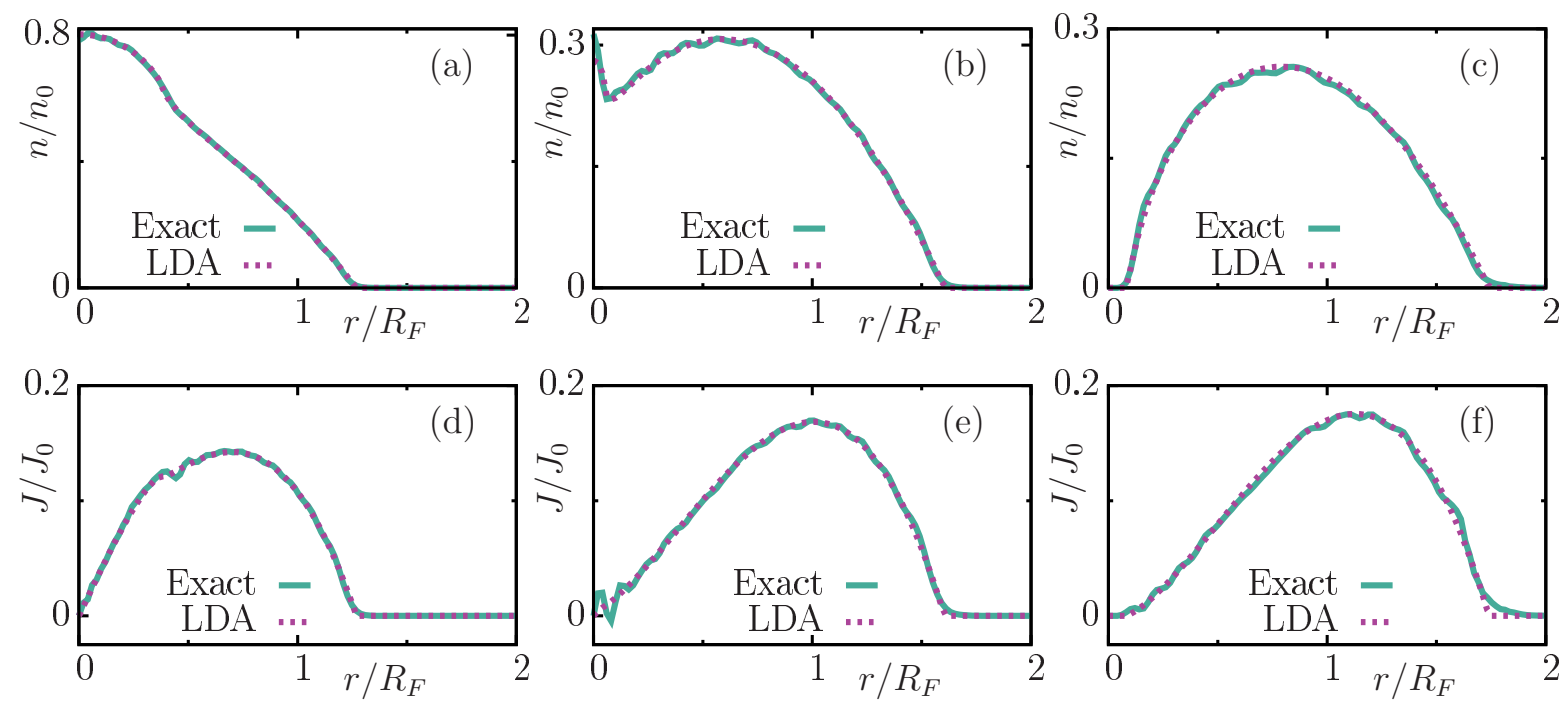

FIG. 6. Exact solutions for the (a)-(c) number density and (d)-(f) mass-current density are compared with those of the semiclassical LDA approach, where $\Omega / \omega=0.5$ in (a) and (d), 0.68 in (b) and (e), and 0.7 in (c) and (f). We also set $N=800$ and $\alpha=1.33 E_{F} / k_{F}$ in all panels. The overall results are in excellent agreement with each other up to minor deviations due to finite-size effects.

[1] J. R. Abo-Shaeer, C. Raman, J. M. Vogels, and W. Ketterle, Science 292, 476 (2001).

[2] M. W. Zwierlein, J. R. Abo-Shaeer, A. Schirotzek, C. H. Schunck, and W. Ketterle, Nature (London) 435, 1047 (2005).

[3] T. L. Ho and C. V. Ciobanu, Phys. Rev. Lett. 85, 4648 (2000).

[4] N. R. Cooper, N. K. Wilkin, and J. M. F. Gunn, Phys. Rev. Lett. 87, 120405 (2001).

[5] U. R. Fischer and G. Baym, Phys. Rev. Lett. 90, 140402 (2003).

[6] M. A. Baranov, K. Osterloh, and M. Lewenstein, Phys. Rev. Lett. 94, 070404 (2005).

[7] M. Z. Hasan and C. L. Kane, Rev. Mod. Phys. 82, 3045 (2010).

[8] X.-L. Qi and S.-C. Zhang, Rev. Mod. Phys. 83, 1057 (2011).

[9] J. Sinova, S. O. Valenzuela, J. Wunderlich, C. H. Back, and T. Jungwirth, Rev. Mod. Phys. 87, 1213 (2015).

[10] I. Žutić and S. Das Sarma, Rev. Mod. Phys. 76, 323 (2004).

[11] Y.-J. Lin, Jiménez-García, and I. B. Spielman, Nature (London) 471, 83 (2011).

[12] J. Y. Zhang, S. C. Ji, Z. Chen, L. Zhang, Z. D. Du, Bo Yan, G. S. Pan, B. Zhao, Y. J. Deng, H. Zhai, S. Chen, and J. W. Pan, Phys. Rev. Lett. 109, 115301 (2012).

[13] P. Wang, Z.-Q. Yu, Z. Fu, J. Miao, L. Huang, S. Chai, H. Zhai, and J. Zhang, Phys. Rev. Lett. 109, 095301 (2012).

[14] L. W. Cheuk, A. T. Sommer, Z. Hadzibabic, T. Yefsah, W. S. Bakr, and M. W. Zwierlein, Phys. Rev. Lett. 109, 095302 (2012).

[15] C. Qu, C. Hamner, M. Gong, C. Zhang, and P. Engels, Phys. Rev. A 88, 021604(R) (2013).

[16] A. J. Olson, S.-J. Wang, R. J. Niffenegger, C.-H. Li, C. H. Greene, and Y. P. Chen, Phys. Rev. A 90, 013616 (2014).

[17] N. Goldman, G. Juzelinas, P. Öhberg, and I. B. Spielman, Rep. Prog. Phys. 77, 126401 (2014).

[18] K. Jiménez-García, L. J. LeBlanc, R. A. Williams, M. C. Beeler, C. Qu, M. Gong, C. Zhang, and I. B. Spielman, Phys. Rev. Lett. 114, 125301 (2015).
[19] L. Huang, Z. Meng, P. Wang, P. Peng, S.-L. Zhang, L. Chen, D. Li, Q. Zhou, and Jing Zhang, Nat. Phys. (to be published).

[20] J. Ruseckas, G. Juzeliūnas, P. Öhberg, and M. Fleischhauer, Phys. Rev. Lett. 95, 010404 (2005).

[21] D. L. Campbell, G. Juzeliūnas, and I. B. Spielman, Phys. Rev. A 84, 025602 (2011).

[22] Z. F. Xu and L. You, Phys. Rev. A 85, 043605 (2012).

[23] J. P. Vyasanakere, S. Zhang, and V. B. Shenoy, Phys. Rev. B 84, 014512 (2011).

[24] Z.-Q. Yu and H. Zhai, Phys. Rev. Lett. 107, 195305 (2011).

[25] M. Iskin and A. L. Subaşı, Phys. Rev. Lett. 107, 050402 (2011).

[26] M. Gong, S. Tewari, and C. Zhang, Phys. Rev. Lett. 107, 195303 (2011).

[27] W. Yi and G.-C. Guo, Phys. Rev. A 84, 031608(R) (2011).

[28] L. Jiang, X.-J. Liu, H. Hu, and H. Pu, Phys. Rev. A 84, 063618 (2011).

[29] K. Zhou and Z. Zhang, Phys. Rev. Lett. 108, 025301 (2012).

[30] R. Liao, Y. Yi-Xiang, and W.-M. Liu, Phys. Rev. Lett. 108, 080406 (2012).

[31] K. Seo, Li Han, and C. A. R. Sá de Melo, Phys. Rev. Lett. 109, 105303 (2012).

[32] S. Takei, C.-H. Lin, B. M. Anderson, and V. Galitski, Phys. Rev. A 85, 023626 (2012).

[33] L. He and X.-G. Huang, Phys. Rev. Lett. 108, 145302 (2012).

[34] M. Gong, G. Chen, S. Jia, and C. Zhang, Phys. Rev. Lett. 109, 105302 (2012).

[35] A. Ambrosetti, G. Lombardi, L. Salasnich, P. L. Silvestrelli, and F. Toigo, Phys. Rev. A 90, 043614 (2014).

[36] X. Yang and S. Wan, Phys. Rev. A 85, 023633 (2012).

[37] W. Zhang and W. Yi, Nat. Commun. 4, 2711 (2013).

[38] M. Iskin, Phys. Rev. A 88, 013631 (2013). 
[39] Y. Cao, S.-H. Zou, X.-J. Liu, S. Yi, G.-L. Long, and H. Hu, Phys Rev. Lett. 113, 115302 (2014).

[40] K. Martiyanov, V. Makhalov, and A. Turlapov, Phys. Rev. Lett. 105, 030404 (2010).

[41] P. Dyke, E. D. Kuhnle, S. Whitlock, H. Hu, M. Mark, S. Hoinka, M. Lingham, P. Hannaford, and C. J. Vale, Phys. Rev. Lett. 106, 105304 (2011).

[42] B. Fröhlich, M. Feld, E. Vogt, M. Koschorreck, W. Zwerger, and M. Köhl, Phys. Rev. Lett. 106, 105301 (2011).

[43] M. Feld, B. Fröhlich, E. Vogt, M. Koschorreck, and M. Köhl, Nature (London) 480, 75 (2011).

[44] A. T. Sommer, L. W. Cheuk, M. J. H. Ku, W. S. Bakr, and M. W. Zwierlein, Phys. Rev. Lett. 108, 045302 (2012).

[45] M. G. Ries, A. N. Wenz, G. Zürn, L. Bayha, I. Boettcher, D. Kedar, P. A. Murthy, M. Neidig, T. Lompe, and S. Jochim, Phys. Rev. Lett. 114, 230401 (2015).
[46] I. Bausmerth, A. Recati, and S. Stringari, Phys. Rev. Lett. 100, 070401 (2008).

[47] I. Bausmerth, A. Recati, and S. Stringari, Phys. Rev. A 78, 063603 (2008).

[48] M. Urban and P. Schuck, Phys. Rev. A 78, 011601 (2008).

[49] M. Iskin and E. Tiesinga, Phys. Rev. A 79, 053621 (2009).

[50] H. J. Warringa and A. Sedrakian, Phys. Rev. A 84, 023609 (2011).

[51] H. J. Warringa, Phys. Rev. A 86, 043615 (2012).

[52] J. Radić, T. A. Sedrakyan, I. B. Spielman, $\begin{array}{lllll}\text { and V. Galitski, Phys. Rev. A 84, } 063604 & \end{array}$ (2011).

[53] M. Stone and I. Anduaga, Ann. Phys. (N.Y.) 323, 2 (2008). 